\title{
El uso educativo de las TIC
}

\author{
Oscar Yecid Aparicio Gómez ${ }^{1}$
}

Recibido: 26-01-2018

Aceptado: 22-05-2018

\section{Resumen}

El presente artículo ${ }^{2}$ pretende describir y analizar los usos de las TIC en una Institución Educativa del Municipio de Chía, y la percepción de los estudiantes y profesores. Este estudio permite el acercamiento y percepción al uso de las TIC en los diversos espacios académicos, su relación con la investigación, el uso de las Plataformas que provee el Colegio en el proceso de aprendizaje, la relación entre las redes sociales y el aprendizaje, algunas percepciones que se tienen sobre las TIC y la necesidad de acceder de manera crítica a la información, la relación entre las TIC y la interdisciplinariedad, el uso que se les da a las TIC para buscar el acercamiento entre la escuela y la familia, y finalmente, se pretende conocer la apreciación de las TIC como herramienta para optimizar el proceso de aprendizaje e investigación.

Palabras clave: transferencia del conocimiento, TIC, instituciones educativas, colegio, investigación, metodología de la investigación, instrumentos de investigación, pedagogía.

1. Doctor en Filosofía (2006) y Doctor en Educación (2015) por la Universidad de Barcelona. Profesor y Editor.

Bogotá - Colombia.

Correo electrónico: oscar.yecid@gmail.com

ORCID: 0000-0003-3535-6288

2. La información puede ampliarse en: Aparicio, Ó. Y. (2015). Las TIC como herramienta cognitiva para la investigación escolar (Tesis doctoral, Universitat de Barcelona, Barcelona España). Recuperado de http://hdl.handle.net/10803/369830 


\title{
The education use of TIC
}

\begin{abstract}
This article aims to describe and analyze the uses of ICT in an Educational Institution of the Municipality of Chia, and the perception of students and teachers. This study allows the approach and perception to the use of the $\mathrm{TIC}$ in the diverse academic spaces, its relation with the investigation, the use of the Platforms that the School provides in the process of learning, the relation between the social networks and the learning, some perceptions about ICTs and the need to critically access information, the relationship between ICT and interdisciplinarity, the use that ICTs are given to seek the approach between school and family, and finally, it is intended to know the appreciation of ICT as a tool to optimize the learning and research process.
\end{abstract}

Keywords: knowledge transfer, TIC, educational institutions, school, research, research methodology, research instruments, pedagogy.

\section{Introducción}

Este cuestionario ha sido elaborado para conocer cómo se usan las TIC en una Institución Educativa del Municipio de Chía de acuerdo con la percepción de los estudiantes y profesores. En razón a sus objetivos, características y contextualización, corresponde a un estudio de casos, de caso único y concreto en su particularidad temporal y local, el cual, como afirma Flick (2012), parte de las expresiones y actividades de las personas en sus contextos locales, es decir, que no se quiere buscar una teoría que explique el porqué del comportamiento de los estudiantes con respecto a las TIC. Simplemente, se quiere conocer la percepción del uso que se les dan a las TIC en el contexto de la educación escolar en una Institución Educativa del Municipio de Chía.

Existen tres razones por las cuales, en coherencia con el marco indicado, hemos optado por el cuestionario como instrumento de esta investigación: 
- La necesidad de recolectar datos para poder hacer el análisis respectivo. El cuestionario es una forma de obtener, de manera sistemática y ordenada, información sobre las variables que intervienen en una investigación (Visauta, 1989).

- La necesidad de conocer la percepción de los estudiantes y profesores. Con el cuestionario la información resultante hace referencia a lo que las personas son, hacen, opinan, sienten, esperan, desean, quieren u odian, aprueban o desaprueban, o los motivos de sus actos, opiniones y actitudes (Visauta, 1989).

- La gran cantidad de personas sobre las que se quiere realizar esta investigación. El cuestionario se debe usar en el caso que sea necesario conocer la opinión de una gran cantidad de personas, es el instrumento más adecuado en esta investigación.

De este modo, los participantes del cuestionario pueden dar a conocer sus percepciones sin tener que limitarse a algunas opciones. Sin embargo, este tipo de cuestionario puede generar ambigüedades en las respuestas, como por ejemplo que los participantes no respondan exactamente a lo que se les pregunta; pero con el objetivo de evitar esta situación se explicó previamente a los encuestados en qué consistían las preguntas según su edad y nivel académico.

\section{Metodología}

Preparada la versión 1.0 del Cuestionario "Uso de las TIC en una Institución Educativa del Municipio de Chía", esta se entregó a los expertos para que revisaran su Validez, Fiabilidad y Pertinencia.

Diseñada y revisada la versión 1.0 de cada instrumento, se entregaron los cuestionarios a un grupo de expertos para que revisaran su Validez, Fiabilidad y Pertinencia, entendidas como:

- Validez de contenido: adecuación y correlación entre lo que se dice que se quiere "medir" o sobre la información 
que se quiere recoger y lo que realmente se "mide" o qué información se recoge. Según Guba (1989: 153) es importante no olvidar la relación entre validez interna y rigor, y validez externa y relevancia o "generalizabilidad".

- Fiabilidad o Confiabilidad: exactitud de la "medición" o de la recogida de información.

- Pertinencia u Objetividad: relevancia y adecuación del instrumento en cuanto a los objetivos del estudio y al grupo de participantes (Judikis, en prensa).

En la perspectiva naturalista interpretativa en la cual nos enmarcamos, podemos asociar estos criterios de credibilidad a los siguientes términos más adecuados al marco naturalista (Guba, 1989, 153): Validez interna con Credibilidad, Validez externa con Transferibilidad, Fiabilidad con Dependencia, y Pertenencia u Objetividad con Confirmabilidad.

Estos criterios fueron valorados con una escala de Likert, llamada así por su autor, Rensis Likert, en la que cada respuesta solo podía estar categorizada en una sola opción, a elegir entre las siguientes cuatro posibilidades o niveles: muy alta, alta, baja, muy baja. Aunque tradicionalmente las escalas de Likert tienen 5 opciones, en este caso hemos incluido solo 4 para que los jueces ajusten su juicio. Además, se les solicitó que añadieran cualquier observación relativa a la redacción, así añadirían algún ítem, la ordenación de estos, etc.

Una vez recibidos los comentarios de los expertos y luego de elaborar a partir de ellas los ajustes sugeridos, se procedió a elaborar la versión 2.0 del Cuestionario "Uso de las TIC en una Institución Educativa del Municipio de Chía", versión definitiva que se entregaría a los destinatarios.

El procedimiento que se ha utilizado para realizar este estudio es a través de la distribución de cuestionarios impresos en papel a la población de muestra, de manera que los pueden resolver en el aula de clase y así obtener los datos inmediatamente. Consideramos importante aclarar que aunque el sentido de la pregunta era la misma o similar para todos, la formulación y orientación sobre las preguntas se adaptó igualmente al nivel educativo de cada uno.

La versión 2.0 del Cuestionario "Uso de las TIC en una Institución Educativa del Municipio de Chía", fue aplicada a estudiantes y profesores, y 
se compone de 10 preguntas. Aunque las preguntas son idénticas para estudiantes y profesores, en el caso de la pregunta número 6, se matizó para aplicarla a los profesores. Las preguntas irán precedidas por el número de Instrumento y número de pregunta correspondiente.

El cuestionario que se les proporcionó a los participantes, con el detaIle del objetivo de cada pregunta fue el siguiente:

01/P1. ¿Qué tan frecuentemente hacen uso los profesores de las TIC en sus clases? Objetivo: Conocer la percepción que tienen los estudiantes sobre la frecuencia de uso que le dan los profesores a las herramientas TIC dentro de las clases.

01/P2. ¿Cuáles son los principales usos que se dan a las TIC en las clases? Objetivo: Reconocer las herramientas TIC que usan los profesores dentro de las clases, teniendo en cuenta la percepción de los estudiantes.

01/P3. ¿Cómo se usan las TIC para investigar? Objetivo: Identificar la percepción que tienen los estudiantes sobre las herramientas TIC de las que hacen uso los profesores para la investigación.

01/P4. ¿Para qué usan la Plataforma Académica digital de la Institución? Objetivo: Averiguar la impresión que tienen los estudiantes acerca del uso que le dan los profesores a la plataforma académica y conocer qué clase de elementos son publicados en esta.

01/P5. ¿Cómo usan habitualmente las redes sociales? Objetivo: Conocer el uso que los estudiantes dan a las redes sociales para las labores académicas, haciendo énfasis en el trabajo colaborativo que se puede lograr con esta clase de herramienta.

01/P6. (Estudiantes) ¿Con qué frecuencia se hacen las publicaciones a través de la Plataforma del Colegio? Objetivo: Apreciar la percepción de los estudiantes sobre la frecuencia con que se realizan las publicaciones a través de la Plataforma del Colegio.

01/P6. (Profesores) ¿Cómo hacen las publicaciones a través de la Plataforma del Colegio? Objetivo: Conocer la manera como 
los profesores realizan las publicaciones a través de la Plataforma del Colegio.

01/P7. ¿Favorece el uso de las TIC el trabajo interdisciplinar? ¿De qué manera? Objetivo: Distinguir lo que piensan los estudiantes sobre la forma y los usos que se les pueden dar a las TIC para favorecer el trabajo interdisciplinario entre diferentes áreas del conocimiento.

01/P8. ¿Considera que se mejora el acceso a la información a través de las TIC? ¿Cómo? Objetivo: Analizar las ventajas que encuentran los estudiantes en el acceso a la información gracias a las herramientas TIC que tienen acceso.

01/P9. ¿Las TIC, a través de Plataformas y redes sociales, favorecen el flujo de comunicación entre la escuela y la familia? ¿De qué manera? Objetivo: Valorar la impresión que tienen los estudiantes sobre las ventajas que se tienen gracias a los nuevos canales de comunicación que han abierto las TIC para favorecen la comunicación entre la escuela y la familia.

01/P10. ¿Percibes que el uso de las TIC mejora el aprendizaje? ¿Cómo? Objetivo: Recoger la percepción de los estudiantes sobre la manera en la que las TIC mejoran el aprendizaje.

\section{Resultados}

A continuación se presenta una comparación entre los resultados arrojados del estudio del cuestionario aplicado a los estudiantes y a los profesores. Las preguntas formuladas son presentadas en la primera columna de la tabla, y las categorías que recogen las preguntas de estudiantes y profesores aparecen en las dos columnas inferiores. Igualmente, las categorías que aparecen en letra "negrita" corresponden a las más significativas de cada colectivo, en letra "cursiva y color similar" aparecen aquellas donde coinciden estudiantes y profesores, y en letra "negrita y cursiva" pueden apreciarse las categorías que son las más significativas para estudiantes y profesores. 


\begin{tabular}{|c|c|}
\hline ESTUDIANTES & PROFESORES \\
\hline $\begin{array}{l}\text { - A: Alto - En más de un } 85 \% \text { de las clases. } \\
\text { - M-A: Medio-Alto - Entre un } 60 \% \text { y un } 85 \% \text { de } \\
\text { las clases. } \\
\text { - M: Medio - Entre un } 30 \% \text { y un } 60 \% \\
\text { - MB: Medio-Bajo - Entre un } 10 \% \text { y un } 30 \% \\
\text { - B: Bajo - Menos del } 10 \% \\
\text { - NP: No Respondió a la Pregunta. } \\
\text { - NR: No Responde. }\end{array}$ & $\begin{array}{l}\text { - A: Alto - En más de un } 85 \% \text { de las clases. } \\
\text { - M-A: Medio-Alto - Entre un } 65 \% \text { y un } 85 \% \text { de } \\
\text { las clases. } \\
\text { - M: Medio - Entre un } 30 \% \text { y un } 60 \% \text { de las } \\
\text { clases. } \\
\text { - MB: Medio-Bajo - Entre un } 5 \% \text { y un } 30 \% \text { de las } \\
\text { clases. } \\
\text { - B: Bajo - Menos del } 5 \% \text { de las clases. }\end{array}$ \\
\hline
\end{tabular}

Tabla 1. ¿Qué tan frecuentemente hacen uso los profesores de las TIC en sus clases? Comparativo de las categorías que agrupan las respuestas de los estudiantes y las categorías que agrupan las respuestas de los profesores.

Es posible ver que, tanto en los resultados de los estudiantes como en los resultados de los profesores, se hace un uso bastante reiterativo de las TIC dentro del aula de clase (Konieczny, 2015). En ambos colectivos la mayoría de las respuestas se agrupan en el rango que supera el oscila entre el $60 \%$ y el $85 \%$. Por lo tanto, es posible concluir que la percepción tanto para los estudiantes como para los profesores es que se hace un uso bastante alto en las clases.

\begin{tabular}{|l|l|}
\hline \multicolumn{1}{|c|}{ ESTUDIANTES } & \multicolumn{1}{c|}{ PROFESORES } \\
\hline - ANH: Aprendizaje de Nuevas Herramientas. & - CC-E: Creación de Contenido - Estudiantes. \\
- CC-E: Creación de Contenido - Estudiantes. & $-I$ : Investigación. \\
- I, CI: Investigación, Consulta de Información. & $-\mathrm{J}$ : Juegos. \\
- JE: Juegos Educativos. & - LV: Uso de Libros Virtuales. \\
- LD: Libros Digitales. & $-P M A-E$ : Proyección de Medios Audiovisuales \\
- NP: No Respondió a la Pregunta. & - Estudiante. \\
- NR: No Responde. & $-P M A-P:$ Proyección de Medios Audiovisuales \\
- PC-RVC: Profundizar Conocimientos - Repaso & - - UC: Uso Colaborativo. En esta categoría se \\
de lo Visto en Clase. & agrupan las respuestas que presentan las \\
- PMA-E: Proyección Medios Audiovisuales - & para la realización de sus trabajos. \\
Estudiantes. & \\
- PMA-P: Proyección Medios Audiovisuales - & \\
Profesor. & \\
- RTA: Realización de Trabajos y Actividades. & \\
\hline
\end{tabular}

Tabla 2. ¿Cuáles son los principales usos que se dan a las TIC en las clases? Comparativo de las categorías que agrupan las respuestas de los estudiantes y las categorías que agrupan las respuestas de los profesores 
Con respecto al uso de las TIC en las clases, la percepción de estudiantes y profesores es que el mayor uso que se da es para la proyección de medios audiovisuales por parte del profesor a los estudiantes (por medio del iPad, el Apple TV y el cañón de proyección). Mientras que la segunda categoría en popularidad para los profesores se refiere a la "CCE: Creación de Contenido - Estudiantes", teniendo en cuenta que para los estudiantes fue la quinta en popularidad. Algunas de las categorías que aparecen en las respuestas de los estudiantes no coinciden con las categorías que agrupan las respuestas de los profesores, tales como la "RTA: Realización de Trabajos y Actividades", "PC-RVC: Profundizar Conocimientos - Repaso de lo Visto en Clase" y el "ANH: Aprendizaje de Nuevas Herramientas". No obstante, en general las categorías resultantes en las respuestas de los estudiantes y las de los profesores suelen concordar.

\begin{tabular}{|l|l|}
\hline \multicolumn{1}{|c|}{ ESTUDIANTES } & \multicolumn{1}{c|}{ PROFESORES } \\
\hline - Al: Apoyo a la Investigación. & - CI/PT: Consulta de Información. \\
- AT: Uso del Aula TIC. & I Profundización en los Temas. \\
- CCI: Consulta y Captación de & - GCIAE: Gran Cantidad de Información al \\
Información. & Alcance de los Estudiantes. \\
- CINC: Consulta de Información por Medios & - ME: Motivación para los Estudiantes. \\
No & - RPI: Realización de Productos de la \\
CONVENCIONALES. & Investigación. \\
- M: Motivación de los estudiantes en el tema & \\
de investigación. & \\
- NP: No Respondió a la Pregunta. & \\
- NR: No Responde. & \\
- PR: Las TIC como Medio de Producción \\
de Resultados de las Investigaciones y \\
compartirlos.
\end{tabular}

Tabla 3. ¿Cómo se usan las TIC para investigar? Comparativo de las categorías que agrupan las respuestas de los estudiantes y las categorías que agrupan las respuestas de los profesores.

Sobre el uso de las TIC para investigar (Aparicio \& Ostos, 2018), la categoría que recogió una mayor cantidad de respuestas tanto de profesores como de estudiantes, es la que indica que el mayor uso que le dan a las TIC para la investigación es para "CCl: Consulta y Captación de Información en la Red". Además, para algunos profesores la "M: 
Motivación" que genera las TIC para la investigación es determinante en los estudiantes, sin embargo, para los estudiantes no es uno de los factores más definitorios dentro de sus respuestas.

\begin{tabular}{|l|l|}
\hline \multicolumn{1}{|c|}{ ESTUDIANTES } & \multicolumn{1}{c|}{ PROFESORES } \\
\hline - AC: Actividades Complementarias. & - AC: Actividades Complementarias. \\
- CCIAC: Complemento de las Clases I & - CC: Construcción de Contenido. \\
Apoyo de las Clases. & - EPF: Estimula la Participación Familiar. \\
- CHSC: Conocer lo que se va a Hacer en las & - MA/MI: Material Audiovisual / Material \\
$\quad$ Siguientes Clases. & Interactivo. \\
- IC: Información Complementaria. & RVC: Repaso de lo Visto en Clase. \\
- JE: Juegos Educativos. & \\
- MA: Material Audiovisual. & \\
- NP: No Respondió a la Pregunta. & \\
- NR: No Responde. & \\
- NU: No se Usa de esa Manera. & \\
- PC: Producción de Contenido. & \\
- RVC: Repasar lo Visto en Clase. & \\
\hline
\end{tabular}

Tabla 4. ¿Para qué usan la Plataforma Académica digital de la Institución? Comparativo de las categorías que agrupan las respuestas de los estudiantes y las categorías que agrupan las respuestas de los profesores.

En cuanto al uso que se le da a la Plataforma Académica es muy interesante notar que tanto estudiantes como profesores concuerdan en las primeras categorías en popularidad. Para los estudiantes la categoría más popular es la que se refiere a la "RVC: Repasar lo Visto en Clase", que también resulta ser la más popular en las categorías de los profesores; al mismo tiempo, la tercera categoría en popularidad dentro de los profesores, la que se refiere al "MA: Material Audiovisual" e interactivo publicado en la Plataforma, es la segunda categoría más popular para los estudiantes. Del mismo modo, es posible notar que las "AC: Actividades Complementarias" que es la categoría más popular para los profesores, junto con la que se refiere a la revisión de lo visto en clase, corresponde a la tercera categoría más popular para los estudiantes cuando se refieren a la Plataforma Académica como complemento y apoyo a las clases. 


\begin{tabular}{|c|c|}
\hline ESTUDIANTES & PROFESORES \\
\hline $\begin{array}{l}\text { - AT: Ayudar a Hacer Trabajos. Agrupa las respuestas sobre las ayudas } \\
\text { en la realización de trabajos. } \\
\text { - CTT: Compartir Tareas y Trabajos con los Compañeros para Realizar } \\
\text { las Actividades en Grupo. } \\
\text { - GCAA: Grupo para Comunicación acerca de Actividades Académicas. } \\
\text { - HC: Herramienta de Comunicación. } \\
\text { - NP: No Respondió a la Pregunta. } \\
\text { - NR: No Responde. } \\
\text { - NU: No se Usan las Redes sociales. } \\
\text { - RD: Resolución de Dudas. } \\
\text { - RTTE: Recordar Tareas, Trabajos, Evaluaciones y las Diferentes } \\
\text { Actividades Académicas. }\end{array}$ & $\begin{array}{l}\text { - ACPP: Actualización } \\
\text { con el Conocimiento } \\
\text { que Poseen Otras } \\
\text { Personas. } \\
\text { - CDI: Comunicación } \\
\text { y Divulgación de } \\
\text { Información. } \\
\text { - CSI: Compartir } \\
\text { y Socializar } \\
\text { Información. } \\
\text { - MNU: Manifiesta No } \\
\text { Hacer Uso de } \\
\text { Redes sociales. }\end{array}$ \\
\hline
\end{tabular}

Tabla 5. ¿Cómo usan habitualmente las redes sociales? Comparativo de las categorías que agrupan las respuestas de los estudiantes y las categorías que agrupan las respuestas de los profesores.

En lo relativo al uso de las redes sociales (Viejo, Cabezas, Martínez, 2013), es interesante ver cómo los estudiantes y sus profesores usan las redes sociales para compartir y buscar ayuda de sus compañeros; los profesores colaboran con y buscan ayuda de otros profesores, entre tanto que los estudiantes colaboran con y buscan ayuda de otros estudiantes. Es muy destacable, en este aspecto, el trabajo entre pares.

\begin{tabular}{|c|c|}
\hline ESTUDIANTES & PROFESORES \\
\hline $\begin{array}{l}\text { - A: Alto - Entre un } 80 \% \text { - } 100 \% \\
\text { - M-A: Medio-Alto - Entre un } \\
60 \% \text { - } 80 \% \\
\text { - M: Medio - Entre un } 40 \% \text { - } \\
60 \% \\
\text { - MB: Medio-Bajo - Entre un } \\
20 \% \text { - } 40 \% \\
\text { - B: Bajo - Menos del } 20 \% \\
\text { - NP: No Respondió a la } \\
\text { Pregunta. } \\
\text { - NR: No Responde. }\end{array}$ & $\begin{array}{l}\text { - CC: Complementando lo que se Hace En Clase. } \\
\text { - CCF: Compartiendo el Conocimiento Adquirido con la Familia. } \\
\text { - IA: Dando Información Adicional sobre los Temas de la Clase. } \\
\text { - PAC: Proponiendo Actividades. } \\
\text { Complementarias sobre los Temas de la Clase. } \\
\text { - RC: Realizar Repaso de los Temas de la Clase. } \\
\text { - TC/PP: La Transferencia se Realiza en Clase / La Plataforma } \\
\text { Profundiza. }\end{array}$ \\
\hline
\end{tabular}

Tabla 6. ¿Con qué frecuencia se hacen las publicaciones a través de la Plataforma del Colegio? (Est.) ¿Cómo hacen tus publicaciones a través de la Plataforma del Colegio? (Prof.). Comparativo de las categorías que agrupan las respuestas de los estudiantes y las categorías que agrupan las respuestas de los profesores. 
En este caso, la pregunta es diferente para estudiantes y profesores. En el caso de los estudiantes, la percepción del uso de la Plataforma de Publicaciones es "alta". El matiz de la pregunta a los profesores permite una interpretación distinta, no solo a la frecuencia del uso de esta plataforma, sino específicamente a la función complementaria que atribuyen a la Plataforma de Publicaciones en cuanto a la profundización que pueden brindar a los estudiantes con respecto a los contenidos desarrollados en las clases (Ortiz, Buitrago, 2017).

\begin{tabular}{|c|c|}
\hline ESTUDIANTES & PROFESORES \\
\hline $\begin{array}{l}\text { - AM/PT: Análisis Multidisciplinar / Profundidad en } \\
\text { los Temas. } \\
\text { - ANH: Aprender Nuevas Herramientas. } \\
\text { - CC: Creación de Contenido. } \\
\text { - DT: Disciplina a la Hora de Usar las TIC. } \\
\text { - EC: Elementos que se pueden Compartir en } \\
\quad \text { Algunas Disciplinas. } \\
\text { - FA: Favorece el Aprendizaje. } \\
\text { - I/CI: Investigación / Consulta de Información. } \\
\text { - M: Motivación. } \\
\text { - NF: No Favorecen en Nada. } \\
\text { - NP: No Respondió a la Pregunta. } \\
\text { - NR: No Responde. } \\
\text { - VI: Variedad de Información. }\end{array}$ & $\begin{array}{l}\text { - ACC: Funcionando como Apoyo al Permitir } \\
\text { Conocimiento. } \\
\text { - ETIOD: Permite Enlazar Temas Informáticos } \\
\text { con Saberes de Otras Disciplinas. } \\
\text { - ME: Motivación para los Estudiantes. } \\
\text { - MTVD: Un Medio Tecnológico puede ser } \\
\text { Usado por Algunas Disciplinas. } \\
\text { - VGD: Realizar un Producto Ayuda a Tener } \\
\text { una Visión Global sobre las Distintas } \\
\text { Disciplinas. }\end{array}$ \\
\hline
\end{tabular}

Tabla 7. ¿Favorece el uso de las TIC el trabajo interdisciplinar? ¿De qué manera? Comparativo de las categorías que agrupan las respuestas de los estudiantes y las categorías que agrupan las respuestas de los profesores.

En lo que se refiere al lugar de las TIC con respecto al favorecimiento de la interdisciplinariedad (Aparicio, 2018), la categoría más reiterada por los profesores fue la que se refiere a que un medio tecnológico puede ser "MTVD: Un Medio Tecnológico puede ser Usado por Algunas Disciplinas", se puede hacer un paralelo entre esta categoría y la que se encuentra en las respuestas de los estudiantes cuando se refieren a los "EC: Elementos que se pueden Compartir en Algunas Disciplinas", aunque el número de 
respuestas que destacan esta categoría es considerablemente menor. De manera inversa es posible notar que si bien la categoría referente al acceso a "VI: Variedad de Información" es determinante para los estudiantes, en los profesores no se formula una categoría similar teniendo en cuenta sus respuestas.

\begin{tabular}{|l|l|}
\hline \multicolumn{1}{|c|}{ ESTUDIANTES } & \multicolumn{1}{c|}{ PROFESORES } \\
\hline - FA: Facilidad de Aprendizaje y Estudio. & $\begin{array}{c}\text { EEIO: Los Estudiantes Estructuren la } \\
\text { Información Obtenida. } \\
\text { - FI: Filtros de Información. } \\
\text { - I/CI: Investigación / Consulta de Información. } \\
\text { - M: Motivación de Modo Diferente. }\end{array}$ \\
- NP: No Respondió a la Pregunta. & $\begin{array}{c}\text { Accede de Manera Crítica. } \\
\text { - MI: Usando las TIC como Medio de } \\
\text { Investigación. }\end{array}$ \\
- NR: No Responde. & \\
- RI: Reflexión sobre la Información. & \\
\end{tabular}

Tabla 8. ¿Considera que se mejora el acceso a la información a través de las TIC? ¿Cómo? Comparativo de las categorías que agrupan las respuestas de los estudiantes y las categorías que agrupan las respuestas de los profesores.

Al comparar el tema sobre la mejora del "acceso a la información a través de las TIC", la categoría más popular para los estudiantes es la referente a que se debe "RI: reflexionar sobre la información" que se encuentra, se puede hacer un paralelo en la segunda categoría más popular dentro de las respuestas de los profesores y es la referente a que: "EEIO: Los Estudiantes Estructuren la Información Obtenida". En ambos casos las TIC se resaltan como una herramienta para mejorar el acceso a la información. La categoría más popular para los profesores, y que fue la segunda más popular para los estudiantes, es aquella que se refiere a que "MI: Usando las TIC como Medio de Investigación" permite acceder mejor a la información, lo cual destaca que se pueda acceder a información con puntos de vista diversos (de la calle, Malaver, Gallego, Rodríguez, Flórez, Saldaña, 2014). Una de las categorías menos populares dentro de las que agrupan las respuestas de los estudiantes y que fue también la menos popular dentro de las respuestas de los profesores es la que se refiere a que la información que le llega a los estudiantes debe pasar por "Fl: Filtros de Información" de lo que los estudiantes tienen acceso y de esta manera puedan obtener información confiable. 


\begin{tabular}{|l|c|}
\hline \multicolumn{1}{|c|}{ ESTUDIANTES } & PROFESORES \\
\hline - APEH: Acercamiento de los Padres a la & $\begin{array}{c}\text { EPH: Acerca a los Padres hacia sus } \\
\text { Hijos, su Proceso Académico y su } \\
\text { Proyecto de Vida. }\end{array}$ \\
- API: Acceso de los Padres a la Información del \\
$\begin{array}{c}\text { Colegio. } \\
\text { - CD: Comunicación más Directa entre Colegio } \\
\text { y Padres de Familia. }\end{array}$ & $\begin{array}{c}\text { - FREA: Por su Facilidad, Rapidez, } \\
\text { Efectividad y Asincronía para Entregar } \\
\text { la Información. }\end{array}$ \\
- NP: No Respondió a la Pregunta. & \\
- NR: No Responde. & \\
- VC: No se Hace. & \\
\hline
\end{tabular}

Tabla 9. ¿Las TIC, a través de Plataformas y redes sociales, favorecen el flujo de comunicación entre la escuela y la familia? ¿De qué manera? Comparativo de las categorías que agrupan las respuestas de los estudiantes y las categorías que agrupan las respuestas de los profesores.

Se puede decir que tanto en las respuestas de los estudiantes como de los profesores se puede encontrar que resaltan las mismas ventajas en la manera en la que a través de las Plataformas y las redes sociales, se favorece el flujo de comunicación entre la escuela y la familia; los estudiantes en las dos categorías más populares resaltan la comunicación más directa entre los padres de familia y el colegio, además del acceso a información del colegio que se da gracias a estos medios de comunicación proporcionados por el colegio, mientras que los profesores, en la categoría con más respuestas agrupadas, resaltan las ventajas de la comunicación gracias a las TIC, como la facilidad, la rapidez, la efectividad, la ubicuidad, entre otras. Para estudiantes y profesores, las TIC ofrecen ciertas características que propician una comunicación más cercana entre la familia y la escuela. La mayoría de ellos creen que estas Plataformas y las redes sociales acercan a los padres a la educación de sus hijos.

\begin{tabular}{|l|l|}
\hline \multicolumn{1}{|c|}{ ESTUDIANTES } & \multicolumn{1}{c|}{ PROFESORES } \\
\hline $\begin{array}{l}\text { - AI/Cl: Apoyo a la Investigación / Consulta de } \\
\text { Información. }\end{array}$ & $\begin{array}{c}\text { - AA/PA: Agilidad en el Acceso / A lo largo } \\
\text { de todo el Proceso de Aprendizaje. }\end{array}$ \\
- CA: Contenidos Adicionales a las Clases. & - AC: Permite Analizar el Contexto. \\
- FAA: Se Facilita y Agiliza el Aprendizaje. & $\begin{array}{c}\text { CC/R: Como un Complemento para las } \\
\text { Clases / Para Repaso. }\end{array}$ \\
- M: Motivación para Aprender. & $-\begin{array}{l}\text { M: Afinidad de las Generaciones Actuales } \\
\text { con la Tecnología, lo cual Genera } \\
\text { Motivación. }\end{array}$ \\
- NP: No Respondió a la Pregunta. & - MU: Optimiza Mucho. \\
- NR: No Responde. & - RP: Permite la Realización de un Producto. \\
\hline
\end{tabular}

Tabla 10. ¿Percibes que el uso de las TIC mejora el aprendizaje? ¿Cómo? Comparativo de las categorías que agrupan las respuestas de los estudiantes y las categorías que agrupan las respuestas de los profesores. 
Las opiniones de estudiantes y profesores sobre el uso de las TIC para mejorar el aprendizaje coinciden en que aparecen en el rol escolar (Langer, 2016) como una "M: Motivación", siendo uno de los factores que más ayuda en el aprendizaje de los estudiantes. En los estudiantes es la categoría más popular y dentro de los profesores es muy significativa. Así mismo, ambos grupos afirman que las TIC ayudan en el aprendizaje como apoyo para las clases estimulando a profesores y estudiantes para que se atrevan a profundizar, repasar y reforzar los temas que se vieron en la clase, se destaca que esta categoría fue la segunda más popular dentro de los estudiantes y muy reiterada por los profesores.

En general se pudo observar que las respuestas de los profesores y de los estudiantes coinciden muy frecuentemente. Uno de los contrastes que más llamaron la atención fue el referente al uso que le dan a las redes sociales ya que se comparte el objetivo de trabajo colaborativo, pero solo con sus pares. Puede afirmarse que la frecuencia de las coincidencias en las opiniones de estudiantes y profesores manifiesta la unidad entre los objetivos trazados por el Proyecto Educativo y la percepción que ambos colectivos tienen de estas directrices generales.

\section{Conclusiones}

El análisis de los resultados practicados, obtenidos e interpretados a 183 estudiantes y 15 profesores, y de su comparación, ha permitido conocer la percepción que tienen los estudiantes y los profesores sobre el uso de las TIC en una Institución Educativa del Municipio de Chía, después de cuatro años de inicio del Centro Educativo a la par con la incorporación de las TIC.

A medida que el grado escolar sube los estudiantes perciben que se hace un uso más frecuente de las TIC en sus clases, y es objetivamente comparable y comprobable. Además, se pudo apreciar que tanto los profesores como los estudiantes manifiestan que se hace un uso frecuente de las TIC en sus clases.

En las clases de los grados superiores se hace un uso más diverso de las TIC, mientras que en los primeros grados se reduce hasta llegar a un único uso para Primer grado. Además, el uso más común que dan los profesores a las TIC en las clases es para proyectar medios audiovisuales (videos, presentaciones, grabaciones, etc.). 
En los primeros grados escolares, los profesores se limitan a usar la Plataforma Académica como un medio para que los estudiantes repasen lo que han visto en las clases; mientras tanto, en los grados superiores los profesores usan la Plataforma Académica como un medio para consolidar y ampliar el contenido las clases.

El uso de las redes sociales como herramientas para el trabajo colaborativo se diversifica a medida que el grado escolar aumenta y se intensifica el uso que se dan a las redes sociales para esta labor, pasando de solo usar las redes sociales como una "Herramienta de Comunicación", en los grados inferiores, a usarlas como herramienta de socialización y colaboración entre algunos integrantes del grupo de clase para desarrollar trabajos o tareas, así como para recordarse unos a otros los compromisos escolares. Por su parte, los profesores usan las redes sociales principalmente para compartir información y para socializar datos mientras se busca la retroalimentación para consolidar conocimientos.

El uso de la Plataforma de Publicaciones es alto por parte de los estudiantes, con un uso superior al $65 \%$. Igualmente, el número de respuestas que se invalidaron fue muy significativo, principalmente por dificultades de comprensión del enunciado y muy probablemente por la falta de acompañamiento de los profesores para brindar la explicación adecuada, excepto en Primer grado y Segundo grado.

Por otra parte, tomando en consideración el interés por conocer cómo se favorece la interdisciplinariedad el uso de las TIC, los estudiantes más pequeños responden de forma positiva ante esta pregunta refiriéndose a que "Favorecen el Aprendizaje" pero no especifican cómo. No obstante, a medida que el grado escolar es mayor, los estudiantes pasan de afirmar que "Favorece el Aprendizaje" a especificar cómo se favorece, para estos grados, especialmente desde Sexto grado a Octavo grado, y toman relevancia las categorías "Elementos Tecnológicos que se pueden Compartir en Algunas Disciplinas" y "Variedad de Información". Se demuestra así que aspectos como compartir información en las diversas disciplinas y los medios tecnológicos (videos, presentaciones, juegos, etc.) favorecen la interdisciplinariedad en el uso de las TIC según el parecer de los estudiantes de Secundaria.

La percepción de los estudiantes sobre cómo se favorece el acceso crítico a la información a través de las TIC, se orienta hacia la necesidad de tener el criterio y los métodos para acceder de manera crítica a la 
información a través de las TIC. La mayoría de los estudiantes de Educación Secundaria y Educación media opinan que la información debe ser filtrada por personas con experiencia, tal como los profesores o padres de familia, Sin embargo, los profesores concuerdan con lo que manifiestan los estudiantes, pero enfocados a que en vez de que sean ellos quienes filtren la información, los estudiantes deben aprender métodos para filtrar y acceder críticamente a la información. Con respecto a la percepción sobre el acceso crítico a la información a través de las TIC, los estudiantes insisten en tener un al acceso libre.

En cuanto a la manera en que las TIC, a través de Plataformas y redes sociales favorecen el flujo de comunicación entre la escuela y la familia, tanto los profesores como los estudiantes destacan las ventajas que dan las comunicaciones de manera general (facilidad, rapidez, ubicuidad, actualización constante). No obstante, la mayoría de los estudiantes resaltan el hecho de que la comunicación mediada por las TIC es más directa entre el Colegio y los padres de familia. También sobresale que para los estudiantes de algunos grados intermedios (Cuarto grado y Sexto grado) lo más importante en cuanto al favorecimiento del flujo de comunicación entre la escuela y la familia es el "Acercamiento de los Padres a la Educación de sus Hijos" gracias a las TIC. Para los profesores resulta igualmente importante, teniendo en cuenta que para la pregunta referente a este tema solo se obtuvieron dos categorías en el caso de los profesores, ambas en este sentido.

Según las respuestas de los estudiantes, las TIC optimizan los procesos de aprendizaje, especialmente por medio de la "Motivación" que generan. Además, ellos opinan que las TIC como herramienta para profundizar, repasar y comprobar lo aprendido en las clases y, la facilidad y agilidad para el aprendizaje son también elementos que perfeccionan el aprendizaje.

En cuanto a los profesores, se destaca que sus respuestas hacen alusión a lo que los estudiantes también responden, según aparece indicado en sus respuestas; esta situación puede reconocerse en la manera como se pueden agrupar las respuestas para su posterior categorización, así como la popularidad de las categorías. Algunas veces, las categorías de los estudiantes y los profesores eran prácticamente las mismas y otras veces la categoría más popular de los estudiantes era la misma categoría más popular de los profesores. 


\section{Referencias bibliográficas}

Aparicio, Ó. Y. (2015). Las TIC como herramienta cognitiva para la investigación escolar (Tesis doctoral, Universitat de Barcelona, Barcelona, España). Recuperado de http://hdl. handle.net/10803/369830

Aparicio, O.Y. (2018). Las TIC como herramientas cognitivas. Revista Interamericana de Investigación, Educación y Pedagogía, RIIEP, 11(1). https://orcid.org/0000-0003-35356288

Aparicio, O.Y., \& Ostos, O.L. (2018). Las TIC como herramientas cognitivas para la investigación. Revista Interamericana de Investigación, Educación y Pedagogía, RIIEP, 11(1). https://orcid.org/0000-0003-3535-6288. https://orcid.org/0000-0002-6477-9872

de la Calle, C. V., Malaver, M. O., Gallego, J. D. M., Rodríguez, M., Flórez, J. C., Henao, C. E. \& Saldaña, R. (2014). Aportes de los doctorados de educación en ciencia, tecnología y sociedad, desde la sistematización de sus investigaciones doctorales científicas y formativas, 2000-2010. Revista Interamericana de Investigación, Educación y Pedagogía, RIIEP, 7(1). DOI: https://doi.org/10.15332/s1657-107X.2014.0001.04

Flick, U. (2012). Introducción a la investigación cualitativa. Madrid: Morata.

Guba, E. G. (1989). Criterios de credibilidad en la investigación naturalista. En: Gimeno, J. y Pérez, A. I. (comps.). La enseñanza: su teoría y su práctica (pp. 148-165). Madrid: Akal.

Judikis, J. C. (en prensa). Usos pedagógicos de Moodle en la Universidad de Magallanes, desde la perspectiva de Académicos. Magallanes: Universidad de Magallanes.

Konieczny, P. (2015). Lorenzo García Aretio: bases, mediaciones y futuro de la educación a distancia en la sociedad digital. Revista Interamericana de Investigación, Educación y Pedagogía, RIIEP, 8(1). DOI: https://doi.org/10.15332/s1657-107X.2015.0001.08

Langer, E. (2016). La construcción de confianza para el estudio de prácticas de resistencia en la escolarización de jóvenes en contextos de pobreza urbana. Revista Interamericana de Investigación, Educación y Pedagogía, RIIEP, 9(2). DOI: https://doi. org/10.22490/25391887.1945

Ortiz, J. G., \& Buitrago, H. (2017). La evaluación en la tradición educativa colombiana. Instrumento de clasificación social. Revista Interamericana de Investigación, Educación y Pedagogía, RIIEP, 10(1), 145-171. DOI: https://doi.org/10.15332/s1657-107X.2148

Viejo, C. M., Cabezas, I. L., \& Martínez, M. D. J. I. (2013). Las redes de académicas en la docencia universitaria. Revista Interamericana de Investigación, Educación y Pedagogía, RIIEP, 6(2). DOI: https://doi.org/10.15332/s1657-107X.2013.0002.03

Visauta, B. (1989). Técnicas de investigación social. Barcelona: PPU.

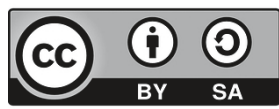

